\title{
The interval between the primary cytoreductive surgery and adjuvant chemotherapy in patients with advanced ovarian cancer
}

\author{
Corresponding author: \\ Mykhailo Chetverikov, Center \\ of Reconstructive and Restorative Medi- \\ cine (University Clinic), 8 Tinista St., \\ Odesa, Ukraine, \\ e-mail: chetvericov@yahoo.com
}

Medical Research Journal 2020; Volume 5, Number 3, 141-147 10.5603/MRJ.a2020.0027 Copyright (C) 2020 Via Medica ISSN 2451-2591

\begin{abstract}
Aim of the study was to establish the effect of the time interval between the initial optimal cytoreductive surgery and the initiation of adjuvant chemotherapeutic treatment (ACT) on the overall survival (OS) of patients with advanced ovarian adenocarcinoma.

Materials and methods. Clinical cases of 60 patients with advanced ovarian adenocarcinoma (FIGO IIIC-IV), with the average age of 61 years, who underwent primary cytoreductive surgery (PDS) with the completeness of cytoreduction (CC) - 0 score according to Shugarbaker and adjuvant chemotherapeutic treatment according to the standard first-line regimen were examined. Patients were categorized depending on the time between surgery and chemotherapeutic treatment into two groups: I — delay of chemotherapy for no more than one month (30 patients), II — from two to six months (30 patients). The OS data of the patients obtained from the national cancer registry were analyzed.

Results. The results demonstrate an increase in OS of patients who underwent CC-O PDS at the early initiation of ACT.

Conclusions. Delaying the onset of ACT is an independent predictor of the worse OS after performing PDS. According to the data obtained, patients should start ACT within 1 month after the surgery. However, the findings are proved if $\mathrm{CC}-0$ is achieved during the operation.

Key words: primary cytoreductive surgery, adjuvant chemotherapeutic treatment, ovarian adenocarcinoma
\end{abstract}

Med Res J 2020; 5 (3): 141-147

\section{Introduction}

According to the National Cancer Registry of Ukraine 2017-2018, ovarian cancer ranks seventh in the structure of the incidence of malignant neoplasms and fifth in the structure of mortality from them among women. Stage III in FIGO had $43.4 \%$, IV - 15.5\% among first identified patients. Nearly $25 \%$ of them did not live for one year. This survival is associated with a predominantly asymptomatic course of the disease in the early stages. Therefore the process is constantly diagnosed in the later stages. Ovarian cancer is classified according to its cellular origin. Most ovarian cancers come from superficial (epithelial) ovarian cells and are called epithelial tumors, although some cancers can also arise from ovarian stroma, from mesenchyme of embryonic gonads, sex stroma, etc. The development of peritoneal carcinomatosis is typical for the most common variant of ovarian cancer (epithelial) due to cellular and molecular processes which are specific to such tumors. It leads to rapid exfoliation of malignant cells into the abdominal cavity. The main methods for the special treatment of epithelial ovarian cancer are surgical and chemotherapeutic. In recent decades the paradigm shift in the treatment of advanced ovarian cancer has been observed. It is accompanied by the introduction of new active methods of combined treatment. According to their pathophysiological properties, these tumors respond relatively well to cytoreductive (surgical and chemotherapeutic) treatment. Any decrease in the total tumor mass has a definite effect on the overall survival (OS) of patients. However, the oncological results of special treatment directly depend on the surgical radicality [1] and the intensity of chemotherapeutic treatment [2]. The size of the residual tumor mass after cytoreductive surgery is an important prognostic factor for the OS of such 
patients. The concept of the cytoreductive intervention was proposed by Paul $\mathrm{H}$. Sugarbaker. It is based on the principle of maximum removal of macroscopically visible implantation metastases from the abdominal cavity in order to achieve the minimum microscopic level of the residual intraperitoneal pool of tumor cells. An increase in surgical radicalness in patients with advanced ovarian cancer is associated with an increase in overall and relapse-free survival. Although, it may lead to prolongation of postoperative recovery and delay the initiation of adjuvant chemotherapy treatment (ACT). The relationship between the interval from surgery and the onset of ACT with overall and relapse-free survival is being investigated. Nevertheless, the optimal interval has not yet been determined. Most studies allow an interval of 6-8 weeks. Until now there remains the issue of the order of combined treatment [3, 4], the volume of resection in standard surgery $[5,6]$ and the total intensity of the combined treatment, i.e., the time interval between each of its stages. Also, the role of hyperthermic intraperitoneal chemoperfusion in the treatment of advanced ovarian cancer still remains without a final assessment $[7,8]$.

Aim of the study was to establish the effect of the time interval between the initial optimal cytoreductive surgery and the initiation of ACT on the OS of patients with advanced ovarian adenocarcinoma.

\section{Materials and methods}

Clinical cases of 60 patients with advanced ovarian adenocarcinoma (FIGO IIIC-IV), with the average age of 61 years (interval from 37 to 71 years), who underwent primary cytoreductive surgery (PDS) with the completeness of cytoreduction (CC) - 0 score according to Shugarbaker and adjuvant chemotherapeutic treatment according to the standard first-line regimen (three-week regimen of carboplatin administration (area under the concentration-time curve 5-6) and paclitaxel $175 \mathrm{mg} / \mathrm{m} 2$ ) were examined. Patients were categorized depending on the time between surgery and chemotherapeutic treatment into two groups: I - delay of chemotherapy for no more than one month (30 patients), II - from two to six months (30 patients). The OS data of the patients obtained from the National Cancer Registry of Ukraine were analyzed. The study was conducted as a part of the scientific work of the Surgery Department No. 4 with a course of oncology at Odessa National Medical University. Compliance with the WMA Code of Ethics of the World Medical Association WMA Declaration of Helsinki - Ethical Principles for Medical Research Involving Human Subjects 2013 (protocol of the meeting of the bioethics commission of the Odessa National Medical University No. 176a of 11/14/2019) was determined. All study participants were informed and agreed to the processing of their clinical data and participation in the research process.

Statistical analysis of the data was carried out using the GNU PSPP program version 1.2.0. Kaplan-Meier survival curves were constructed; the Log Rank test and a special case of the Peto \& Peto modification of the Gehan-Wilcoxon test were used for statistical comparison.

\section{Results}

In the analysis of the first group of patients (the time interval before the start of ACT to 1 month), we obtained a variable series of periods (in months) between the start of special treatment and the establishment of death from the underlying disease and / or its complications: $10,10,12,14,15,15,16,17,18,18,19,19,20,21,22$, $25,26,33,35,39,43,43,43,51,53,56,63,85,101$, 126. Variation indices: weighted average -36 , mode - 43, median -26 , standard deviation -27.513 .

In the analysis of the second group of patients (the time interval before the start of ACT from 2 to 6 months), we obtained a variable series of periods (in months) between the start of special treatment and the establishment of death from the underlying disease and / or its complications: $5,5,5,5,6,6,12,12,12,12,14,14,18$, $18,19,19,19,19,19,19,19,19,19,19,19,27,27,35$, $35,40,40$. Variation indices: weighted average -18 , mode - 19, median - 19, standard deviation - 9.76.

Survival functions and confidence intervals are calculated using the obtained variational series, a table of the lifetime is constructed (Tab. 1.1, 1.2, 2). Kaplan-Meier plots were constructed for a graphical interpretation of the results (Fig. 1). Log Rank criterion was used to test the hypothesis of various survival rates in groups: xi-square 10.485957, $p=0.001203(p<0.05)$, and a special case of the Peto \& Peto modification of the Gehan-Wilcoxon test: xi-square 8.493086, $p=0.003565(p<0.05)$

\section{Discussion of the results}

The results demonstrate an increase in OS of patients who underwent CC-0 PDS with early ACT initiation. Intraoperative seeding by tumor cells is one of the reasons for the growth of residual micrometastases in the interval between surgical resection of the tumor and chemotherapeutic treatment. Such micrometastases are most sensitive to chemotherapeutic treatment. An important factor in the growth of residual tumor mass may be immune suppression in the early postoperative period and the production of pro-inflammatory cytokines. A mechanical effect on the tumor mass causes changes in the cell 
Table 1.1. Group I (<1 month interval)

\begin{tabular}{|c|c|c|c|c|c|c|c|c|c|c|c|}
\hline 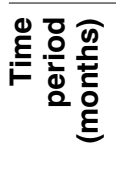 & $\begin{array}{l}\frac{y}{20} \\
\frac{2}{2}\end{array}$ & 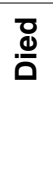 & 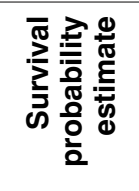 & 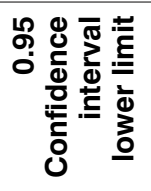 & 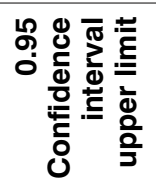 & 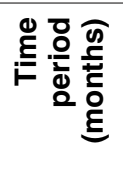 & 市煎 & 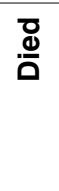 & 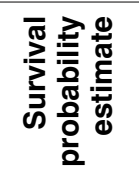 & 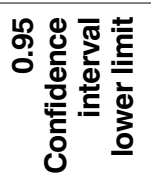 & 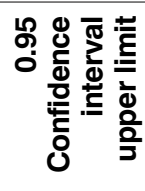 \\
\hline 1 & 30 & 0 & 1 & 0.85868 & 1 & 33 & 13 & 1 & 0.4 & 0.232234 & 0.592497 \\
\hline 2 & 30 & 0 & 1 & 0.85868 & 1 & 34 & 12 & 0 & 0.4 & 0.232234 & 0.592497 \\
\hline 3 & 30 & 0 & 1 & 0.85868 & 1 & 35 & 12 & 1 & 0.366667 & 0.205428 & 0.560919 \\
\hline 4 & 30 & 0 & 1 & 0.85868 & 1 & 36 & 11 & 0 & 0.366667 & 0.205428 & 0.560919 \\
\hline 5 & 30 & 0 & 1 & 0.85868 & 1 & 37 & 11 & 0 & 0.366667 & 0.205428 & 0.560919 \\
\hline 6 & 30 & 0 & 1 & 0.85868 & 1 & 38 & 11 & 0 & 0.366667 & 0.205428 & 0.560919 \\
\hline 7 & 30 & 0 & 1 & 0.85868 & 1 & 39 & 11 & 1 & 0.333333 & 0.179376 & 0.528626 \\
\hline 8 & 30 & 0 & 1 & 0.85868 & 1 & 40 & 10 & 0 & 0.333333 & 0.179376 & 0.528626 \\
\hline 9 & 30 & 0 & 1 & 0.85868 & 1 & 41 & 10 & 0 & 0.333333 & 0.179376 & 0.528626 \\
\hline 10 & 30 & 2 & 0.933333 & 0.764928 & 0.988368 & 42 & 10 & 0 & 0.333333 & 0.179376 & 0.528626 \\
\hline 11 & 28 & 0 & 0.933333 & 0.764928 & 0.988368 & 43 & 10 & 3 & 0.333333 & 0.179376 & 0.528626 \\
\hline 12 & 28 & 1 & 0.9 & 0.723237 & 0.973812 & 44 & 10 & 0 & 0.233333 & 0.10635 & 0.427002 \\
\hline 13 & 27 & 0 & 0.9 & 0.723237 & 0.973812 & 45 & 7 & 0 & 0.233333 & 0.10635 & 0.427002 \\
\hline 14 & 27 & 1 & 0.866667 & 0.683577 & 0.956403 & 46 & 7 & 0 & 0.233333 & 0.10635 & 0.427002 \\
\hline 15 & 26 & 2 & 0.8 & 0.608693 & 0.915952 & 47 & 7 & 0 & 0.233333 & 0.10635 & 0.427002 \\
\hline 16 & 24 & 1 & 0.766667 & 0.572998 & 0.89365 & 48 & 7 & 0 & 0.233333 & 0.10635 & 0.427002 \\
\hline 17 & 23 & 1 & 0.733333 & 0.538273 & 0.870245 & 49 & 7 & 0 & 0.233333 & 0.10635 & 0.427002 \\
\hline 18 & 22 & 2 & 0.666667 & 0.471374 & 0.820624 & 50 & 7 & 0 & 0.233333 & 0.10635 & 0.427002 \\
\hline 19 & 20 & 2 & 0.6 & 0.407503 & 0.767766 & 51 & 7 & 1 & 0.2 & 0.084048 & 0.391307 \\
\hline 20 & 18 & 1 & 0.566667 & 0.376614 & 0.740245 & 52 & 6 & 0 & 0.2 & 0.084048 & 0.391307 \\
\hline 21 & 17 & 1 & 0.533333 & 0.346399 & 0.712034 & 53 & 6 & 1 & 0.166667 & 0.063036 & 0.35451 \\
\hline 22 & 16 & 1 & 0.5 & 0.31685 & 0.68315 & 54 & 5 & 0 & 0.166667 & 0.063036 & 0.35451 \\
\hline 23 & 15 & 0 & 0.5 & 0.31685 & 0.68315 & 55 & 5 & 0 & 0.166667 & 0.063036 & 0.35451 \\
\hline 24 & 15 & 0 & 0.5 & 0.31685 & 0.68315 & 56 & 5 & 1 & 0.133333 & 0.043597 & 0.316423 \\
\hline 25 & 15 & 1 & 0.466667 & 0.287966 & 0.653601 & 57 & 4 & 0 & 0.133333 & 0.043597 & 0.316423 \\
\hline 26 & 14 & 1 & 0.433333 & 0.259755 & 0.623386 & 58 & 4 & 0 & 0.133333 & 0.043597 & 0.316423 \\
\hline 27 & 13 & 0 & 0.433333 & 0.259755 & 0.623386 & 59 & 4 & 0 & 0.133333 & 0.043597 & 0.316423 \\
\hline 28 & 13 & 0 & 0.433333 & 0.259755 & 0.623386 & 60 & 4 & 0 & 0.133333 & 0.043597 & 0.316423 \\
\hline 29 & 13 & 0 & 0.433333 & 0.259755 & 0.623386 & 61 & 4 & 0 & 0.133333 & 0.043597 & 0.316423 \\
\hline 30 & 13 & 0 & 0.433333 & 0.259755 & 0.623386 & 62 & 4 & 0 & 0.133333 & 0.043597 & 0.316423 \\
\hline 31 & 13 & 0 & 0.433333 & 0.259755 & 0.623386 & 63 & 4 & 1 & 0.1 & 0.026188 & 0.276763 \\
\hline 32 & 13 & 0 & 0.433333 & 0.259755 & 0.623386 & 64 & 3 & 0 & 0.1 & 0.026188 & 0.276763 \\
\hline
\end{tabular}

structure, in the microenvironment of tumor cells, as well as changes in their participation in the cell division cycle and the metastatic process. An increase in the amount of mitotically active cells makes them more sensitive to chemotherapeutic drugs that affect the cell division cycle (for example, taxanes). Another factor affecting the increase in the growth rate of residual tumor cells is an increase in the production of angiogenesis factors.
In a study by Tewari K.S. et al. from the materials of phase III of a randomized, double-blind, placebo-controlled trial, the Gynecological Oncology Group protocol 218 obtained similar data on the increase in OS of patients with advanced ovarian cancer with ACT initiation up to 25 days from PDS [9]. Timmermans M. et al. proved that delayed ACT initiation is an independent predictor of OS reduction after cytoreductive surgery, 
Table 1.2. Group I (< 1 month interval)

\begin{tabular}{|c|c|c|c|c|c|c|c|c|c|c|c|}
\hline 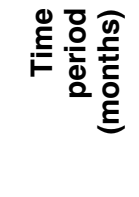 & $\begin{array}{l}\frac{y}{0} \\
\frac{0}{2}\end{array}$ & 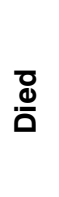 & 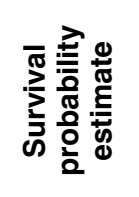 & 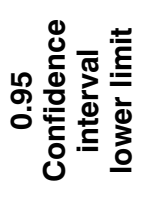 & 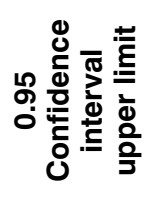 & 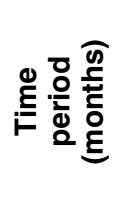 & を竞 & $\frac{\bar{d}}{\frac{\pi}{0}}$ & 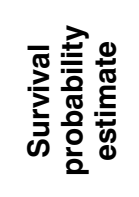 & 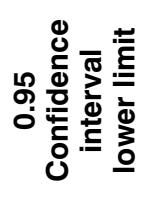 & 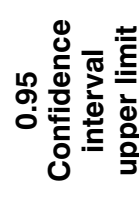 \\
\hline 65 & 3 & 0 & 0.1 & 0.026188 & 0.276763 & 96 & 2 & 0 & 0.066667 & 0.011632 & 0.235072 \\
\hline 66 & 3 & 0 & 0.1 & 0.026188 & 0.276763 & 97 & 2 & 0 & 0.066667 & 0.011632 & 0.235072 \\
\hline 67 & 3 & 0 & 0.1 & 0.026188 & 0.276763 & 98 & 2 & 0 & 0.066667 & 0.011632 & 0.235072 \\
\hline 68 & 3 & 0 & 0.1 & 0.026188 & 0.276763 & 99 & 2 & 0 & 0.066667 & 0.011632 & 0.235072 \\
\hline 69 & 3 & 0 & 0.1 & 0.026188 & 0.276763 & 100 & 2 & 0 & 0.066667 & 0.011632 & 0.235072 \\
\hline 70 & 3 & 0 & 0.1 & 0.026188 & 0.276763 & 101 & 2 & 1 & 0.033333 & 0.001742 & 0.19053 \\
\hline 71 & 3 & 0 & 0.1 & 0.026188 & 0.276763 & 102 & 1 & 0 & 0.033333 & 0.001742 & 0.19053 \\
\hline 72 & 3 & 0 & 0.1 & 0.026188 & 0.276763 & 103 & 1 & 0 & 0.033333 & 0.001742 & 0.19053 \\
\hline 73 & 3 & 0 & 0.1 & 0.026188 & 0.276763 & 104 & 1 & 0 & 0.033333 & 0.001742 & 0.19053 \\
\hline 74 & 3 & 0 & 0.1 & 0.026188 & 0.276763 & 105 & 1 & 0 & 0.033333 & 0.001742 & 0.19053 \\
\hline 75 & 3 & 0 & 0.1 & 0.026188 & 0.276763 & 106 & 1 & 0 & 0.033333 & 0.001742 & 0.19053 \\
\hline 76 & 3 & 0 & 0.1 & 0.026188 & 0.276763 & 107 & 1 & 0 & 0.033333 & 0.001742 & 0.19053 \\
\hline 77 & 3 & 0 & 0.1 & 0.026188 & 0.276763 & 108 & 1 & 0 & 0.033333 & 0.001742 & 0.19053 \\
\hline 78 & 3 & 0 & 0.1 & 0.026188 & 0.276763 & 109 & 1 & 0 & 0.033333 & 0.001742 & 0.19053 \\
\hline 79 & 3 & 0 & 0.1 & 0.026188 & 0.276763 & 110 & 1 & 0 & 0.033333 & 0.001742 & 0.19053 \\
\hline 80 & 3 & 0 & 0.1 & 0.026188 & 0.276763 & 111 & 1 & 0 & 0.033333 & 0.001742 & 0.19053 \\
\hline 81 & 3 & 0 & 0.1 & 0.026188 & 0.276763 & 112 & 1 & 0 & 0.033333 & 0.001742 & 0.19053 \\
\hline 82 & 3 & 0 & 0.1 & 0.026188 & 0.276763 & 113 & 1 & 0 & 0.033333 & 0.001742 & 0.19053 \\
\hline 83 & 3 & 0 & 0.1 & 0.026188 & 0.276763 & 114 & 1 & 0 & 0.033333 & 0.001742 & 0.19053 \\
\hline 84 & 3 & 0 & 0.1 & 0.026188 & 0.276763 & 115 & 1 & 0 & 0.033333 & 0.001742 & 0.19053 \\
\hline 85 & 3 & 1 & 0.066667 & 0.011632 & 0.235072 & 116 & 1 & 0 & 0.033333 & 0.001742 & 0.19053 \\
\hline 86 & 2 & 0 & 0.066667 & 0.011632 & 0.235072 & 117 & 1 & 0 & 0.033333 & 0.001742 & 0.19053 \\
\hline 87 & 2 & 0 & 0.066667 & 0.011632 & 0.235072 & 118 & 1 & 0 & 0.033333 & 0.001742 & 0.19053 \\
\hline 88 & 2 & 0 & 0.066667 & 0.011632 & 0.235072 & 119 & 1 & 0 & 0.033333 & 0.001742 & 0.19053 \\
\hline 89 & 2 & 0 & 0.066667 & 0.011632 & 0.235072 & 120 & 1 & 0 & 0.033333 & 0.001742 & 0.19053 \\
\hline 90 & 2 & 0 & 0.066667 & 0.011632 & 0.235072 & 121 & 1 & 0 & 0.033333 & 0.001742 & 0.19053 \\
\hline 91 & 2 & 0 & 0.066667 & 0.011632 & 0.235072 & 122 & 1 & 0 & 0.033333 & 0.001742 & 0.19053 \\
\hline 92 & 2 & 0 & 0.066667 & 0.011632 & 0.235072 & 123 & 1 & 0 & 0.033333 & 0.001742 & 0.19053 \\
\hline 93 & 2 & 0 & 0.066667 & 0.011632 & 0.235072 & 124 & 1 & 0 & 0.033333 & 0.001742 & 0.19053 \\
\hline 94 & 2 & 0 & 0.066667 & 0.011632 & 0.235072 & 125 & 1 & 0 & 0.033333 & 0.001742 & 0.19053 \\
\hline 95 & 2 & 0 & 0.066667 & 0.011632 & 0.235072 & 126 & 1 & 1 & 0 & 0 & 0.14132 \\
\hline
\end{tabular}


Table 2. Group II (2-6 months interval)

\begin{tabular}{|c|c|c|c|c|c|}
\hline $\begin{array}{l}\text { Time } \\
\text { period (months) }\end{array}$ & At risk & Died & $\begin{array}{c}\text { Survival } \\
\text { probability } \\
\text { estimate }\end{array}$ & $\begin{array}{c}0.95 \\
\text { Confidence } \\
\text { interval lower limit }\end{array}$ & $\begin{array}{c}0.95 \text { Confidence } \\
\text { interval upper } \\
\text { limit }\end{array}$ \\
\hline 1 & 30 & 0 & 1 & 0.85868 & 1 \\
\hline 2 & 30 & 0 & 1 & 0.85868 & 1 \\
\hline 3 & 30 & 0 & 1 & 0.85868 & 1 \\
\hline 4 & 30 & 0 & 1 & 0.85868 & 1 \\
\hline 5 & 30 & 4 & 0.866667 & 0.683577 & 0.956403 \\
\hline 6 & 26 & 2 & 0.8 & 0.608693 & 0.915952 \\
\hline 7 & 24 & 0 & 0.8 & 0.608693 & 0.915952 \\
\hline 8 & 24 & 0 & 0.8 & 0.608693 & 0.915952 \\
\hline 9 & 24 & 0 & 0.8 & 0.608693 & 0.915952 \\
\hline 10 & 24 & 0 & 0.8 & 0.608693 & 0.915952 \\
\hline 11 & 24 & 0 & 0.8 & 0.608693 & 0.915952 \\
\hline 12 & 24 & 4 & 0.666667 & 0.471374 & 0.820624 \\
\hline 13 & 20 & 0 & 0.666667 & 0.471374 & 0.820624 \\
\hline 14 & 20 & 2 & 0.6 & 0.407503 & 0.767766 \\
\hline 15 & 18 & 0 & 0.6 & 0.407503 & 0.767766 \\
\hline 16 & 18 & 0 & 0.6 & 0.407503 & 0.767766 \\
\hline 17 & 18 & 0 & 0.6 & 0.407503 & 0.767766 \\
\hline 18 & 18 & 2 & 0.533333 & 0.346399 & 0.712034 \\
\hline 19 & 16 & 10 & 0.2 & 0.084048 & 0.391307 \\
\hline 20 & 6 & 0 & 0.2 & 0.084048 & 0.391307 \\
\hline 21 & 6 & 0 & 0.2 & 0.084048 & 0.391307 \\
\hline 22 & 6 & 0 & 0.2 & 0.084048 & 0.391307 \\
\hline 23 & 6 & 0 & 0.2 & 0.084048 & 0.391307 \\
\hline 24 & 6 & 0 & 0.2 & 0.084048 & 0.391307 \\
\hline 25 & 6 & 0 & 0.2 & 0.084048 & 0.391307 \\
\hline 26 & 6 & 0 & 0.2 & 0.084048 & 0.391307 \\
\hline 27 & 6 & 2 & 0.133333 & 0.043597 & 0.316423 \\
\hline 28 & 4 & 0 & 0.133333 & 0.043597 & 0.316423 \\
\hline 29 & 4 & 0 & 0.133333 & 0.043597 & 0.316423 \\
\hline 30 & 4 & 0 & 0.133333 & 0.043597 & 0.316423 \\
\hline 31 & 4 & 0 & 0.133333 & 0.043597 & 0.316423 \\
\hline 32 & 4 & 0 & 0.133333 & 0.043597 & 0.316423 \\
\hline 33 & 4 & 0 & 0.133333 & 0.043597 & 0.316423 \\
\hline 34 & 4 & 0 & 0.133333 & 0.043597 & 0.316423 \\
\hline 35 & 4 & 2 & 0.066667 & 0.011632 & 0.235072 \\
\hline 36 & 2 & 0 & 0.066667 & 0.011632 & 0.235072 \\
\hline 37 & 2 & 0 & 0.066667 & 0.011632 & 0.235072 \\
\hline 38 & 2 & 0 & 0.066667 & 0.011632 & 0.235072 \\
\hline 39 & 2 & 0 & 0.066667 & 0.011632 & 0.235072 \\
\hline 40 & 2 & 2 & 0 & 0 & 0.14132 \\
\hline 41 & 0 & 0 & 0 & 0 & 0 \\
\hline
\end{tabular}




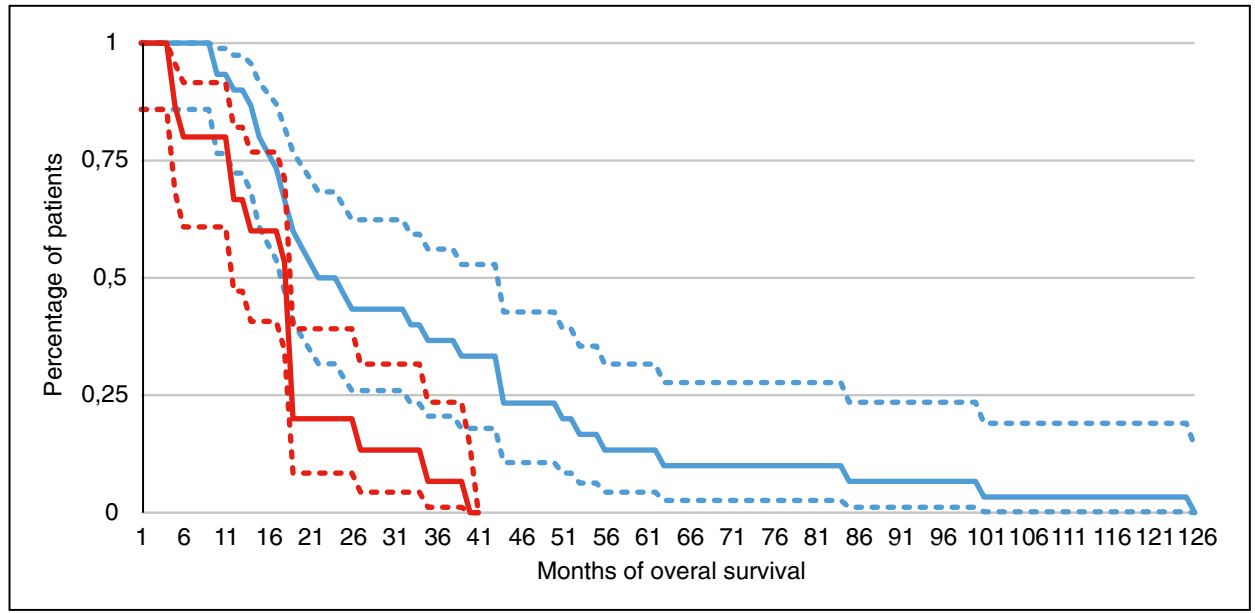

Figure 1. The distribution graphs of the survival of patients I - blue graph (the time interval before the start of ACT to 1 month) and II — red graph (the time interval before the start of ACT from 2 to 6 months) of the Kaplan-Mayer groups with the 0.95 confidence interval shown by dotted lines

and determined the optimal interval of 5-6 weeks [10]. Seagle B.L. et al. performed a retrospective cohort study in patients who underwent special treatment according to the National Comprehensive Cancer Network guideline for 1998-2011 and determined an improvement in survival after ACT initiation within 21-35 days from PDS [11]. In a study by Joseph N. et al., patients with advanced ovarian cancer in the age group of 65 years were considered. Such patients quite often need to reduce the doses of $\mathrm{ACT}$, as well as to delay its initiation. The postponement of ACT is determined by an independent factor associated with a decrease in OS [12]. Similar results were also obtained by Liu Y. et al. in a meta-analysis of 14 studies with 59.569 patients with ovarian cancer. A decrease in OS with a prolonged interval prior to initiation of ACT was found, especially among patients with advanced ovarian cancer [13]. Lee Y. Y. et al. also noted a negative effect on OS with an increase in the intervals between the stages of special treatment of advanced epithelial ovarian cancer [14].

A similar effect is also exerted by a decrease in time between ACT cycles, which was reviewed by Starbuck K.D. et al. In their results, even short delays in the passage of all cycles lead to a progressive decrease in OS [15].

According to Olawaiye A.B. et al. another factor is dose modification in ACT regimens with carboplatin and paclitaxel. Dose reduction and delayed admission increases the risk of disease progression and reduces the OS of patients with advanced ovarian cancer [16].

The results of the Garcia-Soto A.E. et al. study are contradictory. There was demonstrated the lack of effect of time before initiation of adjuvant intraperitoneal chemotherapy on relapse-free survival and OS [17].

Lee Y.J. et al. noted the effect on OS of the time interval from completion of neoadjuvant chemother- apeutic treatment to initiation of ACT in patients with ovarian cancer [18].

In a study, Chen M. et al. demonstrated a decrease in relapse-free survival over a time interval from completion of neoadjuvant chemotherapeutic treatment to cytoreductive surgery for more than 4 weeks, and the absence of the effect of this interval on OS [19].

Jeong S.J et al. noted the absence of a decrease in OS with delayed ACT after secondary cytoreductive surgery due to the progression of ovarian cancer disease [20].

Important prognostic factors for delaying the initiation of chemotherapeutic treatment in patients were postoperative complications, which were often caused by the most aggressive surgical approach to achieve $\mathrm{CC}-0$. It resulted in long periods of stay in a surgical hospital. The treatment opportunities of the oncology center have a great influence on the possibilities of early initiation of ACT because they determine the period of postoperative recovery of the patient and the increase in options for further special treatment of residual disease.

\section{Study Limitations}

This study was a single center, retrospective and nonrandomized. The histological subtypes of ovarian adenocarcinoma of each patient, the degree of differentiation of the tumors, and the Ki67 proliferation index, which probably had a slight effect on the homogeneity of the patient samples, were not taken into account when the groups were formed.

\section{Prospects for further research}

The study of the effect of the interval between neoadjuvant chemotherapeutic treatment and cytoreductive 
surgery is also promising. Comparison of the effectiveness of neoadjuvant chemotherapeutic treatment and further cytoreductive surgery with PDS and adjuvant chemotherapeutic treatment at different intervals between the stages of each of the combined treatment regimens, assessing the quality of life of patients with different options and different treatment intensities is also relevant.

\section{Conclusions}

The results demonstrate a statistically significant difference in the overall survival of patients with advanced ovarian adenocarcinoma (FIGO IIIC-IV) who underwent optimal PDS and ACT according to the standard regimen in a time interval of up to one month compared to the group of patients with a delay of the second stage of treatment by 2-6 months. Thus, delaying the onset of $\mathrm{ACT}$ is an independent predictor of the worse OS after performing PDS. The results of our study highlight the importance of minimizing delays before starting adjuvant chemotherapy. According to the data obtained, patients should start ACT within 1 month after surgery, which is predictive in achieving CC-O in PDS.

\section{Conflict of interest: The authors declare that there is no conflict of interest regarding the publication of this article.}

\section{Abbreviations:}

$$
\begin{aligned}
& \text { ACT - adjuvant chemotherapeutic treatment } \\
& \text { OS - overall survival } \\
& \text { PDS - primary cytoreductive surgery } \\
& \text { CC - completeness of cytoreduction }
\end{aligned}
$$

\section{References}

1. Martinez A, Ngo C, Leblanc E, et al. Surgical Complexity Impac on Survival After Complete Cytoreductive Surgery for Advanced Ovarian Cancer. Ann Surg Oncol. 2016; 23(8): 2515-2521, doi: 10.1245/s10434-015-5069-z, indexed in Pubmed: 27008588.

2. Xu X, Deng F, Lv M, et al. The number of cycles of neoadjuvant chemotherapy is associated with prognosis of stage IIIc-IV high-grade serous ovarian cancer. Arch Gynecol Obstet. 2017; 295(2): 451-458, doi: 10.1007/s00404-016-4256-x, indexed in Pubmed: 27913927.

3. Xiao Y, Xie S, Zhang N, et al. Platinum-Based Neoadjuvant Chemotherapy versus Primary Surgery in Ovarian Carcinoma International Federation of Gynecology and Obstetrics Stages IIIc and IV: A Systematic Review and Meta-Analysis. Gynecol Obstet Invest. 2018; 83(3): 209-219, doi: 10.1159/000485618, indexed in Pubmed: 29402804

4. Kobal B, Noventa M, Cvjeticanin B, et al. Primary debulking surgery versus primary neoadjuvant chemotherapy for high grade advanced stage ovarian cancer: comparison of survivals. Radiol Oncol. 2018; 52(3): 307-319, doi: 10.2478/raon-2018-0030, indexed in Pubmed: 30210049.

5. Somashekhar SP. Does debulking of enlarged positive lymph nodes improve survival in different gynaecological cancers? Best Pract Res Clin Obstet Gynaecol. 2015; 29(6): 870-883, doi: 10.1016/j.bpobgyn.2015.04.010, indexed in Pubmed: 26043964

6. Sinukumar S, Rajan F, Mehta S, et al. A comparison of outcomes following total and selective peritonectomy performed at the time of interval cytoreductive surgery for advanced serous epithelial ovarian, fallopian tube and primary peritoneal cancer - A study by INDEPSO. Eur J Surg Oncol. 2019 [Epub ahead of print], doi: 10.1016/j. ejso.2019.02.031, indexed in Pubmed: 30857879.

7. Zhang G, Zhu Y, Liu C, et al. The prognosis impact of hyperthermic intraperitoneal chemotherapy (HIPEC) plus cytoreductive surgery (CRS) in advanced ovarian cancer: the meta-analysis. J Ovarian Res. 2019; 12(1) 33, doi: 10.1186/s13048-019-0509-1, indexed in Pubmed: 30995948.

8. Le Saux O, Decullier E, Freyer $\mathrm{G}$, et al. Long-term survival in patients with epithelial ovarian cancer following cytoreductive surgery and hyperthermic intraperitoneal chemotherapy (HIPEC). Int J Hyperthermia. 2018; 35(1): 652-657, doi: 10.1080/02656736.2018.1518544, indexed in Pubmed: 30295114

9. Tewari KS, Java JJ, Eskander RN, et al. Early initiation of chemotherapy following complete resection of advanced ovarian cancer associated with improved survival: NRG Oncology/Gynecologic Oncology Group study. Ann Oncol. 2016; 27(1): 114-121, doi: 10.1093/annonc/mdv500, indexed in Pubmed: 26487588

10. Timmermans $M$, van der Aa MA, Lalisang RI, et al. Interval between debulking surgery and adjuvant chemotherapy is associated with overall survival in patients with advanced ovarian cancer. Gynecol Oncol. 2018; 150(3): 446-450, doi: 10.1016/j.ygyno.2018.07.004, indexed in Pubmed: 30001834

11. Seagle BLL, Butler SK, Strohl AE, et al. Chemotherapy delay after primary debulking surgery for ovarian cancer. Gynecol Oncol. 2017; 144(2): 260-265, doi: 10.1016/j.ygyno.2016.11.022, indexed in Pubmed: 27908531

12. Joseph N, Clark RM, Dizon DS, et al. Delay in chemotherapy administration impacts survival in elderly patients with epithelial ovarian cancer. Gynecol Oncol. 2015; 137(3): 401-405, doi: 10.1016/j. ygyno.2015.03.052, indexed in Pubmed: 25839911.

13. Liu Yi, Zhang T, Wu Q, et al. Relationship between initiation time of adjuvant chemotherapy and survival in ovarian cancer patients: a dose-response meta-analysis of cohort studies. Sci Rep. 2017; 7(1): 9461, doi: 10.1038/s41598-017-10197-1, indexed in Pubmed: 28842667.

14. Lee YY, Lee JW, Lu L, et al. Impact of interval from primary cytoreductive surgery to initiation of adjuvant chemotherapy in advanced epithelial ovarian cancer. Int J Gynaecol Obstet. 2018; 143(3): 325-332, doi: 10.1002/ijgo.12653, indexed in Pubmed: 30129040.

15. Starbuck KD, Szender JB, Duncan WD, et al. Prognostic impact of adjuvant chemotherapy treatment intensity for ovarian cancer. PLoS One. 2018; 13(11): e0206913, doi: 10.1371/journal.pone.0206913, indexed in Pubmed: 30418985.

16. Olawaiye AB, Java JJ, Krivak TC, et al. Does adjuvant chemotherapy dose modification have an impact on the outcome of patients diagnosed with advanced stage ovarian cancer? An NRG Oncology/Gynecologic Oncology Group study. Gynecol Oncol. 2018; 151(1): 18-23, doi: 10.1016/j.ygyno.2018.07.021, indexed in Pubmed: 30135020

17. Garcia-Soto AE, Java JJ, Nieves NeiraW, et al. Does time interval between surgery and intraperitoneal chemotherapy administration in advanced ovarian cancer carry a prognostic impact? An NRG Oncology/Gynecologic Oncology Group study ancillary study. Gynecol Oncol. 2016; 143(3): 484489, doi: 10.1016/j.ygyno.2016.10.003, indexed in Pubmed: 27726923.

18. Lee YJ, Chung YS, Lee JY, et al Impact of the time interval from completion of neoadjuvant chemotherapy to initiation of postoperative adjuvant chemotherapy on the survival of patients with advanced ovarian cancer. Gynecol Oncol. 2018; 148(1): 62-67, doi: 10.1016/j. ygyno.2017.11.023, indexed in Pubmed: 29174056.

19. Chen M, Chen Z, Xu M, et al. Impact of the Time Interval from Neoadjuvant Chemotherapy to Surgery in Primary Ovarian, Tubal, and Peritoneal Cancer Patients. J Cancer. 2018; 9(21): 4087-4091, doi: 10.7150/jca.26631, indexed in Pubmed: 30410613.

20. Jeong SY, Choi CH, Kim TJ, et al. Interval between secondary cytoreductive surgery and adjuvant chemotherapy is not associated with survivals in patients with recurrent ovarian cancer. J Ovarian Res. 2019; 13(1): 1, doi: 10.1186/s13048-019-0602-5, indexed in Pubmed: 31892329. 